\title{
Locally hermitian partial ovoids of unitary polar spaces and partial ovoids of orthogonal polar spaces
}

full screen

close

quit

\author{
Alessandro Siciliano
}

\begin{abstract}
In order to study unitals in the projective plane $\mathrm{PG}\left(2, q^{2}\right)$, F. Buekenhout [5] gave a representation in $\mathrm{PG}(4, q)$ of the unitary polar space $H\left(2, q^{2}\right)$ as points of a quadratic cone on a $Q^{-}(3, q)$.

In [16], G. Lunardon used the Barlotti-Cofman representation of PG $\left(3, q^{2}\right)$ to represent $H\left(3, q^{2}\right)$ in $\mathrm{PG}(6, q)$ as a cone on a $Q^{+}(5, q)$. He also proved that to any locally hermitian ovoid of $H\left(3, q^{2}\right)$ corresponds an ovoid of $Q^{+}(5, q)$ and conversely.

In this paper, we study the Barlotti-Cofman representation of the unitary polar space $H\left(n, q^{2}\right)$ for all $n$ and we prove that to any locally hermitian partial ovoid of such spaces corresponds a partial ovoid of an orthogonal polar space, and conversely. Further the locally hermitian partial ovoid is maximal if and only if the corresponding partial ovoid of the orthogonal polar space is maximal. As a consequence of the previous connection and a result of A. Klein [14] we obtain a geometric proof to derive that the orthogonal polar space $Q^{+}(4 n+1, q)$ has no ovoid when $n>q^{3}$.
\end{abstract}

Keywords: polar spaces, partial ovoids, ovoids

MSC 2000: 05B25, 51E20

\section{Introduction}

In the projective space $\mathrm{PG}(n, q)$ coordinatized by the finite field $\mathrm{GF}(q)$ let $\mathcal{P}$ denote a classical polar space. The generators of $\mathcal{P}$ are the subspaces of maximal dimension contained in it. 





Now let $\mathcal{O}$ be a locally hermitian partial ovoid of $H\left(n, q^{2}\right)$ with respect to $(\mathbf{x}, \mathbf{0})$ consisting of $s$ hyperbolic lines. In $S\left(\Sigma^{\prime}, \Sigma, \mathcal{N}_{\Sigma}\right)$ the ovoid $\mathcal{O}$ is represented by the union of $s$ lines of $\mathcal{K}$ containing $V$ one of which is $\ell(\mathbf{x})$. Note that each of these lines but not $\ell(\mathbf{x})$ has $q$ points in $\mathcal{K} \backslash \Sigma$. Hence there is a set $\mathbb{O}$ of $s+1$ points of $Q^{-}(2 n-1, q)$ such that $\langle V, X\rangle, X \in \mathbb{O}$, are the $s+1$ lines through $V$ representing $\mathcal{O}$.

Theorem 3.1. The set $\mathbb{O}$ is a partial ovoid of $Q^{-}(2 n-1, q)$ containing $R=$ $\ell(\mathbf{x}) \cap \Delta$.

Proof. Let $X, Y$ be two distinct points of $\mathbb{O}$ and suppose that $X$ and $Y$ are collinear in $Q^{-}(2 n-1, q)$.

Let $m$ be the line of $\mathcal{N}_{\Sigma}$ incident with the point $\langle X, Y\rangle \cap \Sigma$. If $m=\ell(\mathbf{x})$ then the plane $\langle\ell(\mathbf{x}), X, Y\rangle$ is a plane of the cone $\mathcal{K}$ which represents a line of $H\left(n, q^{2}\right)$ through $(\mathbf{x}, \mathbf{0})$ not in $\alpha$. But this is a contradiction. Hence $m \neq \ell(\mathbf{x})$. Let $S$ be the 3-dimensional subspace $\langle\ell(\mathbf{x}), m\rangle$ and $\mathcal{S}$ the regular line-spread induced by $\mathcal{N}$ in $S$.

Thus the 4-dimensional subspace $\langle S, X\rangle$ represents a plane $\pi$ which is tangent to $H\left(n, q^{2}\right)$ at a point represented by a line of $\mathcal{S}$ and containing the point $(\mathbf{x}, \mathbf{0})$ and the two points of $H\left(n, q^{2}\right)$ represented by $X$ and $Y$. But this is impossible because the plane generated by two hyperbolic lines of $\mathcal{O}$ through $(\mathbf{x}, \mathbf{0})$ is never a tangent plane of $H\left(n, q^{2}\right)$.

Theorem 3.2. Let $\mathbb{O}$ be a partial ovoid of $Q^{-}(2 n-1, q)$ of size $s+1$ containing $R=\ell(\mathbf{x}) \cap \Delta$. Let $\overline{\mathbb{O}}$ be the set of lines $\langle P, X\rangle$, with $X \in \mathbb{O}$. Then $\overline{\mathbb{O}}$ represents a locally hermitian partial ovoid $\mathcal{O}$ with respect to $(\mathbf{x}, \mathbf{0})$ of size $s q+1$.

Proof. We can argue as in [16, Theorem 7].

When $n$ is odd we can argue as before to prove the following result.

Theorem 3.3. Let $\mathcal{O}$ be a locally hermitian partial ovoid of $H\left(n, q^{2}\right)$, $n$ odd, with respect to $(\mathbf{x}, \mathbf{0})$ consisting of $s$ hyperbolic lines. The set $\mathbb{O}$ defined as before is a partial ovoid of $Q^{+}(2 n-1, q)$ of size $s+1$ containing $R=\ell(\mathbf{x}) \cap \Delta$. Conversely, if $\mathbb{O}$ is a partial ovoid of $Q^{+}(2 n-1, q)$ of size $s+1$ containing $R=\ell(\mathbf{x}) \cap \Delta$ then there exists a locally hermitian partial ovoid $\mathcal{O}$ with respect to $(\mathbf{x}, \mathbf{0})$ of size $s q+1$.

Corollary 3.4. The unitary polar space $H\left(n, q^{2}\right)$ has no locally hermitian ovoid when $n$ is odd, $n \geq 5$ and $q=2,3$.

Proof. By way of contradiction the result follows since $Q^{+}(2 n+1, q)$ has no ovoids when $n \geq 4$ and $q=2,3[13,23]$. 


[4] R. H. Bruck, Construction problems in finite projective spaces, Combinatorial Mathematics and its Applications, North Carolina Press, Chapell Hill, 1969, 426-514.

[5] F. Buekenhout, Existence of unitals in finite translation planes of order $q^{2}$ with kernel of order $q$, Geom. Dedicata 5 (1976), 189-194.

[6] J. H. Conway, P. B. Kleidman and R. Wilson, New families of ovoids in $\mathrm{O}_{8}^{+}$, Geom. Dedicata 26 (1988), 157-170.

full screen

close

quit

[7] B. N. Cooperstein, A sporadic ovoid in $\Omega^{+}(8,5)$ and some nonDesarguesian translation planes of order 25, J. Combin. Theory Ser. A 54 (1990), 135-140.

[8] A. Cossidente, G. L. Ebert, G. Marino and A. Siciliano, Shult sets and translation ovoids of the Hermitian surface, Adv. Geom. 6 (2006), 523542.

[9] J. De Beule, A. Klein, K. Metsch and L. Storme, Partial ovoids and partial spreads in symplectic and orthogonal polar spaces, European $J$. Combin., to appear.

[10] J. De Beule and K. Metsch, The Hermitian variety $H(5,4)$ has no ovoid, Bull. Belg. Math. Soc. Simon Stevin 12 (2005), 727-733.

[11] A. Gunawardena, G. E. Moorhouse, The non-existence of ovoids of $O_{9}(q)$, European J. Combin. 18 (1997), 171-173.

[12] W. M. Kantor, Ovoids and translation planes, Canad. J. Math. 34 (1982), 1195-1207.

[13] _ Spreads, translation planes and Kerdock sets, I, Siam. J. Alg. Disc. Methods 3 (1982), 151-165.

[14] A. Klein, Partial ovoids in classical finite polar spaces, Des. Codes Cryptogr. 31 (2004), 221-226.

[15] G. Lunardon, Normal spreads, Geom. Dedicata 75 (1999), 245-261.

[16] _ Blocking sets and semifields, J. Combin. Theory Ser. A, 113 (2006), 1172-1188.

[17] G. Lunardon and O. Polverino, Translation ovoids of orthogonal polar spaces, Forum Math. 16 (2004), 663-669.

[18] F. Mazzocca, O. Polverino and L. Storme, Blocking sets in PG $\left(r, q^{n}\right)$, Des. Codes Cryptogr. 44 (2007), 97-113. 
